\title{
How the lignin-first biorefinery strategy unfolded
}

\author{
Bert Sels ${ }^{1}$, Sander Van den Bosch ${ }^{1}$, Wouter Schutyser ${ }^{1}$, Tom Renders ${ }^{1}$, and Backstories \\ $\operatorname{Admin}^{2}$ \\ ${ }^{1}$ Center for Surface Chemistry and Catalysis, KU Leuven, Kasteelpark Arenberg 23, 3001 \\ Heverlee, Belgium \\ ${ }^{2}$ Backstories
}

January 5, 2018

\begin{abstract}
This article is the backstory behind: S. Van den Bosch, W. Schutyser, B.F. Sels, et al., Reductive lignocellulose fractionation into soluble lignin-derived phenolic monomers and dimers and processable carbohydrate pulps (Van den Bosch et al., 2015).
\end{abstract}

Today, alternative technologies are being developed to drastically reduce our society's dependency on fossil resources. In this light, the conversion of biomass to chemicals is one important objective. Interestingly, recent studies on European forest management have ensured the possibility for a durable production of lignocellulosic biomass (e.g. wood, grass), in quantities that will be sufficient for the replacement of all fossil-based chemicals. This off course requires the development of suitable technology.

The publication demonstrates an innovative biorefinery strategy in which both carbohydrate and lignin components, present in lignocellulose feedstocks (e.g. wood, grass) are valorised into a handful of high-value chemicals. This stands in strong contrast with traditional biorefinery processes, like paper mills, where the lignin side-stream is typically regarded as waste and burned for the production of heat and electricity. Since the lignin biopolymer represents the largest renewable source of aromatics, its conversion to (aromatic) chemicals would however greatly benefit the sustainability and economic viability of future lignocellulosic biorefineries.

In the context of valorising lignin towards chemicals, PhD-student Wouter Schutyser and his master thesis student Sander Van den Bosch started working on the catalytic conversion of lignin model compounds back in 2012. After several successful results, they shifted their attention to the valorisation of relevant isolated lignin streams, such as lignins from paper mills, but soon experienced the discrepancy between simple model compounds and real, complex lignin substrates. Only very low product yields, i.e. lignin monomers, were obtained from real lignin substrates, which was attributed to the 'degraded' lignin structure, obtained after traditionally applied isolation procedures. Refusing to participate in the (at the time) existing paradigm that "you can make anything out of lignin, except money", it was clear that a more ground-breaking solution was needed.

In 2013, their curiosity was triggered by a few articles, which illustrated the more susceptible nature of native lignin, i.e. lignin as present in lignocellulosic biomass, compared to isolated lignins, for the reductive conversion (depolymerisation) towards aromatic chemicals (Yan et al., 2008; Song et al., 2013; Bower et al., 1943; Pepper et al., 1951). Instead of processing isolated lignins, they therefore attempted to use raw lignocellulose substrates such as birch, poplar, pine, and miscanthus grass as feedstocks in their process, and directly convert the native lignin into lignin monomers. Although sceptical at first, a spectacular improvement in the obtained lignin monomer yields and selectivities was observed after the first reactions on lignocellulose 


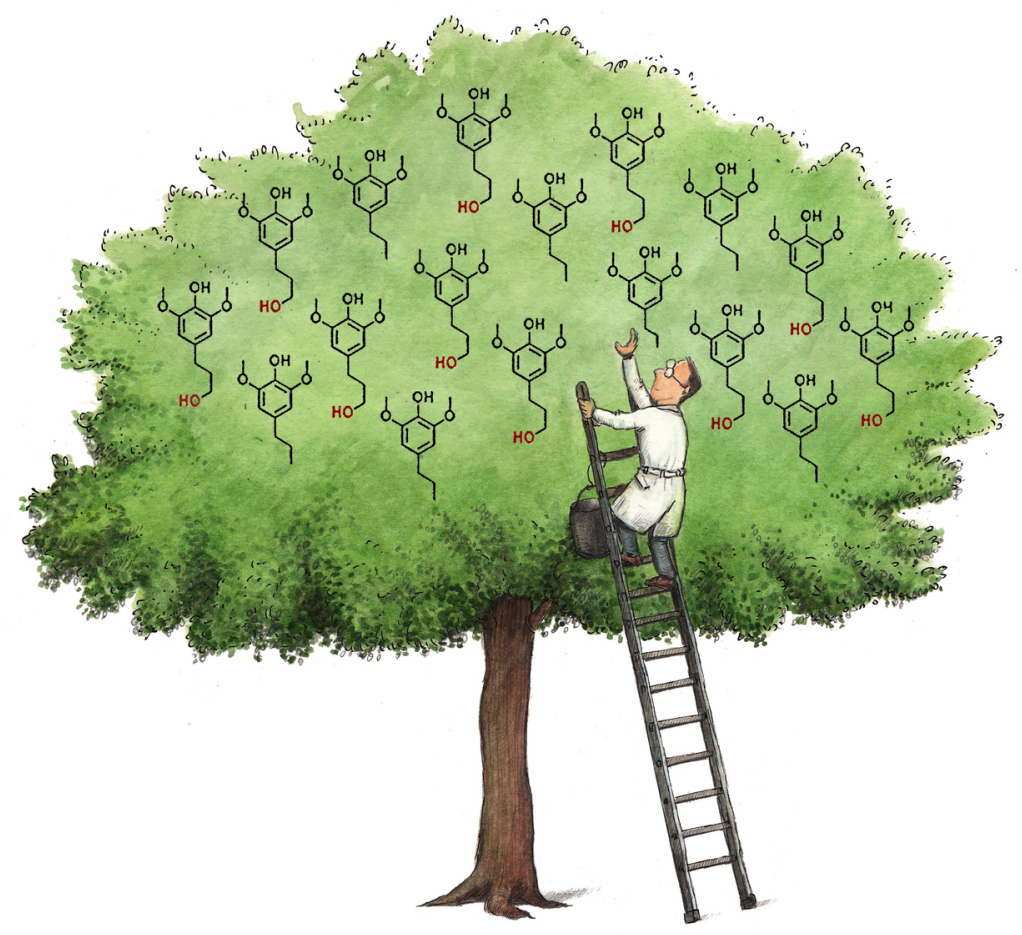

feedstocks. It was later established that a crucial process step is the reductive stabilisation of reactive lignin intermediates, which is essential to prevent degradation reactions and associated low product yields. Instead of only focusing on the lignin monomers, a lot of effort was put in the structural characterisation of the entire lignin product fraction by GC-MS, 2D-NMR and GPC, which revealed interesting structural features in the dimers and oligomers as well. Furthermore, the valorisation potential of the delignified carbohydrate pulp towards sugar alcohols was demonstrated, thus achieving a complete valorisation of lignocellulose's major constituents.

The applied strategy was promoted as a lignin-first strategy, since lignin valorisation is prioritised at an early stage of the biorefinery process, when lignin is still in its most reactive form (Renders et al., 2017). Interestingly, the lignin-first principle is now gaining momentum and will hopefully generate a paradigm shift within the field of lignocellulosic biorefining (Renders et al., 2017).

In their research group, the article formed a kick-start for further investigations which led to an improved understanding and control over the process. To optimise the fractionation efficiency as well as to mitigate the temperature and process conditions, the influence of the solvent (Schutyser et al., 2015; Renders et al., 2016a) and the implementation of additives (H3PO4, NaOH)(Renders et al., 2016b) were studied. Moreover, the elucidation of the catalyst function, regarding lignin solubilisation, depolymerisation and stabilisation, provided new insights that will create opportunities for future reactor design and upscaling (Van den Bosch et al., 2017). At this moment, this technology is successfully performed at $2 \mathrm{~L}$ scale and the produced lignin oils are subjected to application testing, in collaboration with industry and other universities in the frame of multiple projects, i.e. BIOWOOD, BIO-HArT, BAFTA, MAIA, ARBOREF, BIOCON,... 


\section{References}

J.R. Bower, L. M. Cooke, and H. Hibbert. Studies on Lignin and Related Compounds. LXX. Hydrogenolysis and Hydrogenation of Maple Wood. Journal of the American Chemical Society, 65(6):1192-1195, jun 1943. doi: 10.1021/ja01246a049. URL https://doi.org/10.1021\%2Fja01246a049.

J. M. Pepper, C. J. Brounstein, and D. A. Shearer. Studies on Lignin by Means of Catalytic Hydrogenation of Aspen Wood and Wheat Straw1,2. Journal of the American Chemical Society, 73(7):3316-3319, jul 1951. doi: 10.1021/ja01151a095. URL https://doi.org/10.1021\%2Fja01151a095.

T. Renders, S. Van den Bosch, T. Vangeel, T. Ennaert, S.F. Koelewijn, G. Van den Bossche, C. M. Courtin, W. Schutyser, and B. F. Sels. Synergetic Effects of Alcohol/Water Mixing on the Catalytic Reductive Fractionation of Poplar Wood. ACS Sustainable Chemistry \&S Engineering, 4(12):6894-6904, oct 2016a. doi: 10.1021/acssuschemeng.6b01844. URL https://doi.org/10.1021\%2Facssuschemeng. 6b01844.

T. Renders, W. Schutyser, S. Van den Bosch, S.F. Koelewijn, T. Vangeel, C. M. Courtin, and B.F. Sels. Influence of Acidic (H3PO4) and Alkaline $(\mathrm{NaOH})$ Additives on the Catalytic Reductive Fractionation of Lignocellulose. ACS Catalysis, 6(3):2055-2066, feb 2016b. doi: 10.1021/acscatal.5b02906. URL https: //doi.org/10.1021\%2Facscatal.5b02906.

T. Renders, S. Van den Bosch, S.-F. Koelewijn, W. Schutyser, and B. F. Sels. Lignin-first biomass fractionation: the advent of active stabilisation strategies. Energy 85 Environmental Science, 10(7):1551-1557, 2017. doi: 10.1039/c7ee01298e. URL https://doi.org/10.1039\%2Fc7ee01298e.

W. Schutyser, S. Van den Bosch, T. Renders, T. De Boe, S.-F. Koelewijn, A. Dewaele, T. Ennaert, O. Verkinderen, B. Goderis, C. M. Courtin, and B. F. Sels. Influence of bio-based solvents on the catalytic reductive fractionation of birch wood. Green Chemistry, 17(11):5035-5045, 2015. doi: 10.1039/c5gc01442e. URL https://doi.org/10.1039\%2Fc5gc01442e.

Q. Song, F. Wang, J.Cai, Y. Wang, J. Zhang, W. Yu, and J. Xu. Lignin depolymerization (LDP) in alcohol over nickel-based catalysts via a fragmentation-hydrogenolysis process. Energy 63 Environmental Science, 6(3):994, 2013. doi: 10.1039/c2ee23741e. URL https://doi.org/10.1039\%2Fc2ee23741e.

S. Van den Bosch, W. Schutyser, R. Vanholme, T. Driessen, S.-F. Koelewijn, T. Renders, B. De Meester, W. J. J. Huijgen, W. Dehaen, C. M. Courtin, B. Lagrain, W. Boerjan, and B. F. Sels. Reductive lignocellulose fractionation into soluble lignin-derived phenolic monomers and dimers and processable carbohydrate pulps. Energy \& Environmental Science, 8(6):1748-1763, 2015. doi: 10.1039/c5ee00204d. URL https://doi.org/10.1039\%2Fc5ee00204d.

S. Van den Bosch, T. Renders, S. Kennis, S.-F. Koelewijn, G. Van den Bossche, T. Vangeel, A. Deneyer, D. Depuydt, C. M. Courtin, J. M. Thevelein, W. Schutyser, and B. F. Sels. Integrating lignin valorization and bio-ethanol production: on the role of $\mathrm{Ni}-\mathrm{Al} 2 \mathrm{O} 3$ catalyst pellets during lignin-first fractionation. Green Chemistry, 19(14):3313-3326, 2017. doi: 10.1039/c7gc01324h. URL https://doi.org/10.1039\% 2Fc7gc01324h.

N. Yan, C. Zhao, P. J. Dyson, C. Wang, L. Liu, and Y. Kou. Selective Degradation of Wood Lignin over Noble-Metal Catalysts in a Two-Step Process. ChemSusChem, 1(7):626-629, jul 2008. doi: 10.1002/cssc. 200800080. URL https://doi.org/10.1002\%2Fcssc. 200800080. 